\title{
A canine model of Alzheimer's disease generated by overexpressing a mutated human amyloid precursor protein
}

\author{
GEUN-SHIK LEE ${ }^{1 *}$, YEON WOO JEONG ${ }^{2,7 *}$, JOUNG JOO KIM ${ }^{2}$, SUN WOO PARK $^{2}$, KYEONG HEE KO $^{2}$, \\ MINA KANG ${ }^{2}$, YU KYUNG KIM ${ }^{2}$, EUI-MAN JUNG ${ }^{3}$, CHANGJONG MOON $^{4}$, SANG HWAN HYUN $^{5}$, \\ KYU-CHAN HWANG ${ }^{2}$, NAM-HYUNG KIM ${ }^{6}$, TAEYOUNG SHIN ${ }^{2}$, EUI-BAE JEUNG ${ }^{3}$ and WOO SUK HWANG ${ }^{2}$ \\ ${ }^{1}$ College of Veterinary Medicine and Institute of Veterinary Science, Kangwon National University, Chucheon, \\ Gangwon 200-701; ${ }^{2}$ SooAm Biotech Research Foundation, Seoul 152-904; ${ }^{3}$ Laboratory of Veterinary Biochemistry and \\ Molecular Biology, College of Veterinary Medicine, Chungbuk National University, Cheongju, Chungbuk 361-763; \\ ${ }^{4}$ Department of Veterinary Anatomy, College of Veterinary Medical Institute, Chonnam National University, Gwangju, \\ Gyeonggi 500-757; ${ }^{5}$ Laboratory of Veterinary Embryology and Biotechnology, College of Veterinary Medicine, \\ ${ }^{6}$ Department of Animal Science, Chungbuk National University, Cheongju, Chungbuk 361-763; \\ ${ }^{7}$ College of Veterinary Medicine, Seoul National University, Seoul 151-742, Republic of Korea
}

Received September 17, 2013; Accepted January 22, 2014

DOI: $10.3892 /$ ijmm.2014.1636

\begin{abstract}
Canines are considered the most authentic model for studying multifactorial human diseases, as these animals typically share a common environment with man. Somatic cell nuclear transfer (SCNT) technology along with genetic engineering of nuclear donor cells provides a unique opportunity for examining human diseases using transgenic canines. In the present study, we generated transgenic canines that overexpressed the human amyloid precursor protein $(A P P)$ gene containing well-characterized familial Alzheimer's disease (AD) mutations. We successfully obtained five out of six live puppies by SCNT. This was confirmed by observing the expression of green fluorescence protein in the body as a visual transgenic marker and the overexpression of the mutated $A P P$ gene in the brain. The transgenic canines developed AD-like symptoms, such as enlarged ventricles, an atrophied hippocampus, and $\beta$-amyloid plaques in the brain. Thus, the transgenic canines we created can serve as a novel animal model for studying human AD.
\end{abstract}

Correspondence to: Professor Woo-Suk Hwang, SooAm Biotech Research Foundation, San 43-41 Oryu-dong, Guro-gu, Seoul 152-904, Republic of Korea

E-mail: hwangws@sooam.org

Professor Eui-Bae Jeung, Laboratory of Veterinary Biochemistry and Molecular Biology, College of Veterinary Medicine, Chungbuk National University, Cheongju, Chungbuk 361-763, Republic of Korea E-mail: ebjeung@cbu.ac.kr

*Contributed equally

Key words: disease model, amyloid precursor protein, somatic cell nuclear transfer, transgenic canines

\section{Introduction}

Alzheimer's disease (AD) is a devastating neurodegenerative disorder that usually affects individuals of an older age (1). Human life expectancy has increased tremendously over the years due to improved public health programs and advances in clinical medicine. At the same time, the incidence of $\mathrm{AD}$ is rapidly increasing. It has been reported that $>15$ million individuals are suffering from $\mathrm{AD}$ and this number is expected to more than double in the next generation (2). Studies on the pathogenesis of $\mathrm{AD}$ require the use of animal models that develop some degree of amyloid pathologies in the brain (3). The utilization of animal models is crucial for any biomedical research on human disease processes at the cellular and molecular levels, and for developing novel therapies. A transgenic animal model may also be used to examine the pathogenic mechanisms of $\mathrm{AD}$ and related disorders, such as frontotemporal dementia. Furthermore, these models may aid in the development of vaccines or improve current treatment strategies.

$\mathrm{AD}$ is considered a multifactorial and polygenic disease in which environmental and genetic factors play a major role. Although several candidate genes have been surveyed, three are believed to be responsible for the rare early-onset familial form of the disease. These include the amyloid precursor protein $(A P P)$, as well as the presenilin 1 (PSENI) and presenilin 2 (PSEN2) genes (1). Genetically modified mice, flies, fish and worms have been developed that reproduce different $A D$ histopathologies, such as plaques containing $\beta$-amyloid (A $\beta$ ) and tau-containing neurofibrillary tangles (NFTs) (4). Previusly, the production of transgenic mini-pigs carrying the APP695sw transgene using a handmade cloning procedure was reported (4). However, to our knowledge, AD-like symptoms in a transgenic canine model have not been published to date.

One main purpose of an animal model of $\mathrm{AD}$ is to replicate the symptoms, lesions, or causes of the disease. Numerous transgenic murine lines have successfully been used to 
partially reproduce $\mathrm{AD}$ lesions, such as extracellular deposits of the $A \beta$ peptide and the intracellular accumulation of the tau protein (5). Mutated human APP (mhAPP) transgenes result in the deposition of the $A \beta$ peptide, similar but not identical to the plaques observed in senile humans (6). Canines are extensively used in various biomedical studies as model animals. The development of successful somatic cell nuclear transfer (SCNT) procedures in canines has made considerable progress. Consequently, small, medium and large breed dogs have been cloned from cultured cells as the genetic donor or long-term cryopreserved somatic cells (5,7-9). Therefore, it has become possible to use donor cells to create knockout and knockin canines or animals overexpressing the gene of interest. In the present study, we established a somatic cell line overexpressing $m h A P P$ to generate a canine model of AD by SCNT. The transgenic canines were confirmed to have an increased $m h A P P$ expression in the brain, resulting in the accumulation of $A \beta$, enlarged ventricles, an atrophied hippocampus and abnormal behavior.

\section{Materials and methods}

Animal care and welfare. Standard procedures established by the SooAm Biotech Research Foundation (Seoul, Korea) for the Accreditation of Laboratory Animal Care were followed strictly when caring for the dogs used in our study. All experiments and surgical procedures were conducted in accordance with the Guidelines for the Care and Use of Laboratory Animals published by the SooAm Biotech Research Foundation. Canines at the pro-estrus stage (mixed breed, 1-7 years old, 20-25 kg body weight) were obtained from a breeder and reared indoors in separated cages up until oocyte recovery. Following oocyte recovery, the canines were sent back to the breeder. None of the dogs were used repeatedly. The pregnant recipient canines were kept in the research facility while the others were returned to the breeder.

Chemicals. All chemicals were purchased from Sigma-Aldrich (St. Louis, MO, USA), unless otherwise stated.

Cell culture and establishment of canine fibroblasts. Human IMR-32 neuroblastoma cells (Korean Cell Line Bank, Seoul, Korea) were maintained in Dulbecco's modified Eagle's medium (DMEM) containing penicillin and streptomycin, and $10 \%$ fetal bovine serum (FBS) (all from Invitrogen, Carlsbad, CA, USA). Primary culture of canine adult and fetal fibroblasts was performed as described in a previous study (8). Briefly, fetal fibroblasts were obtained from artificially inseminated embryos on the 30th day of pregnance by trypsinization. Fibroblasts were cultured in DMEM containing 10\% FBS.

Construction of transgenic vector. Various regions of the Thy-1 promoter [from nucleotides -2.0 to +2.3 kilonucleotides (knts), $+1=$ the transcriptional start site $]$ were isolated by a long-range polymerase chain reaction (PCR) (LA Taq; Takara Bio, Inc., Shiga, Japan) using genomic DNA from beagle fibroblasts as a template. Amplified fragments were ligated into the $M l u \mathrm{I}$ site of the promoterless pGL3-Basic vector (Promega, Madison, WI, USA). Human APP cDNA was prepared by PCR using the full-length clone from the Mammalian Gene Collection (cat. no. 6152423; Invitrogen) as a template. The amplified APP gene was mutated by two primer sets containing Swedish mutation (KM to NL) or Indiana mutation (V to F) indicated in Table I using a Site-Directed Mutagenesis kit (Stratagene, Inc., La Jolla, CA, USA). The mutated APP gene were further ligated into the $N c o \mathrm{I}$ and $\mathrm{XbaI}$ sites of the recombinant pGL3-Basic vector containing the Thy-1 promoter. An enhanced green fluorescent protein (EGFP) gene from the internal ribosomal entry site (pIRES)2_EGFP (Clontech Laboratories, Inc., Madison, WI, USA) was ligated into the EcoRV and BamHI sites of the pIRES_Neo vector (Clontech Laboratories, Inc.). This selection cassette was inserted into the SalI site of the backbone plasmid containing the dog Thy-1 promoter region and mutant human APP gene. The final construct (pGL3-pThy1mhAPP-pCMV-EGFP/Neo) was linearized by NotI digestion (Fig. 1). All amplified products obtained at each step of the construct production were analyzed by sequencing analysis (Genotech Co. Ltd., Daejeon, Korea).

Transient transfection and reporter gene assay. To control for different transfection efficiencies of the various luciferase constructs, a Rous sarcoma virus (RSV)-lacZ plasmid was used to co-transfect the IMR-32 cells along with the Thy- 1 promoterluciferase construct. Briefly, 3x105 IMR-32 cells were seeded in six-well tissue culture plates one day prior to transfection. Thy- 1 promoter-luciferase constructs $(3.5 \mu \mathrm{g})$ and $0.5 \mu \mathrm{g}$ of the RSV-lacZ plasmid were used to transiently transfect the cells using Lipofectamine ${ }^{\mathrm{TM}} 2000$ (Invitrogen) followed by incubation for an additional $48 \mathrm{~h}$. Cellular lysates were prepared using $150 \mu 1$ of reporter lysis buffer and assayed for luciferase activity using the Luciferase Assay System (both from Promega). Luminescence was measured using a GloMax 20/20 Luminometer (Promega) and $\beta$-galactosidase activity was measured using a $\beta$-galactosidase Enzyme Assay System (Promega). Promoter activity was expressed as a percentage of relative luciferase activity (RLA \%; luciferase/ $\beta$-galactosidase activity).

Laparotomy and oocyte collection. Mature canine oocytes were collected in vivo by laparotomy at 72-84 h after ovulation using standard procedures $(8,9)$. The time of ovulation was determined by the serum progesterone concentration and vaginal cytology (8). All the operative and post-operative procedures and care were performed by licensed veterinarians. The reproductive tract was exposed during mid-ventral laparotomy under a general anesthesia. The fimbriated end of the oviduct was canalized using a six-gauge bulbed needle held in place by a surgical ligature. The oviductal lumen at the base of the utero-oviductal junction was cannulated using a 24-gauge hypodermic needle (Angiocath TM Plus; BectonDickinson, Franklin Lakes, NJ, USA). Approximately $10 \mathrm{ml}$ of TCM 199 collection medium supplemented with HEPES (Sigma-Aldrich) was used to flush the hypodermic needle. The medium passed through the oviductal lumen and bulbed needle before being deposited into a sterile plastic Petri dish. After flushing both oviducts, each ovarian bursa was incised to pull the ovary out and the corpora lutea were counted. Finally, the abdominal incision was closed using a two-layer method followed by the application of a surgical adhesive along the skin incision. 
Table I. Primer sequences.

\begin{tabular}{|c|c|c|}
\hline Name & Direction & Sequences $\left(5^{\prime}\right.$ to $\left.3^{\prime}\right)$ \\
\hline Human $A P P$ cDNA & $\begin{array}{l}\text { Forward } \\
\text { Reverse }\end{array}$ & $\begin{array}{l}\text { CGC GCA GGG TCG CGA TGC TGC CCG } \\
\text { CTG TGG CGG GGG TCT AGT TCT GCA }\end{array}$ \\
\hline Thy- 1 promoter region & $\begin{array}{l}\text { Forward } \\
\text { Reverse }\end{array}$ & $\begin{array}{l}\text { GAC TGG ACC ATC CTT GCA GCT CAT } \\
\text { GCT CAG TCC TTG ATC TGG GGG TGG }\end{array}$ \\
\hline KM to NL (Swedish mutation) & $\begin{array}{l}\text { Forward } \\
\text { Reverse }\end{array}$ & $\begin{array}{l}\text { CGA GAT TCT GAA GTG AAC CTG GAT GCA GAA TTC CGA CAT G } \\
\text { CAT GTC GGA ATT CTG CAT CCA GGT TCA CTT CAG AAT CTC G }\end{array}$ \\
\hline V to F (Indiana mutation) & $\begin{array}{l}\text { Forward } \\
\text { Reverse }\end{array}$ & $\begin{array}{l}\text { GGA CTC ATG GTG GGC GGT TTT GTC ATA GCG ACA GTG ATC } \\
\text { GAT CAC TGT CGC TAT GAC AAA ACC GCC CAC CAT GAG TCC }\end{array}$ \\
\hline EGFP & $\begin{array}{l}\text { Forward } \\
\text { Reverse }\end{array}$ & $\begin{array}{l}\text { CAC AAC CAT GGT GAG CAA GGG CGA } \\
\text { TTA CTT GTA CAG CTC GTC CAT GCC }\end{array}$ \\
\hline $\begin{array}{l}\text { Confirming primer } \mathrm{A} \\
\text { Confirming primer } \mathrm{B}\end{array}$ & $\begin{array}{l}\text { Forward } \\
\text { Reverse }\end{array}$ & $\begin{array}{l}\text { CCT TGT GCT GTC TCC CCC TC } \\
\text { TCA CAA AGT GGG GAT GGG TC }\end{array}$ \\
\hline $\begin{array}{l}\text { Confirming primer } \mathrm{C} \\
\text { Confirming primer } \mathrm{D}\end{array}$ & $\begin{array}{l}\text { Forward } \\
\text { Reverse }\end{array}$ & $\begin{array}{l}\text { CAT GAA GCA GCA CGA CTT CT } \\
\text { CCT AGG AAT GCT CGT CAA GA }\end{array}$ \\
\hline Confirming primer for human $A P P$ & $\begin{array}{l}\text { Forward } \\
\text { Reverse }\end{array}$ & $\begin{array}{l}\text { GAG GTG GAA GAA GAA GAA GCC } \\
\text { GTC AAC GGC ATC AGG GGT ACT }\end{array}$ \\
\hline Confirming primer for canine $A P P$ & $\begin{array}{l}\text { Forward } \\
\text { Reverse }\end{array}$ & $\begin{array}{l}\text { GAT GTT GAG GAA GAG GAA GCT GAG G } \\
\text { GGG AAG AGG TTC CTG GGT TG }\end{array}$ \\
\hline
\end{tabular}

Proper restriction enzyme sequences were added to the 5' region. KM to NL represents Swedish (K670N and M671L) mutations and V to F represents Indiana FAD (V717F) mutations. APP, amyloid precursor protein; EGFP, enhanced green fluorescent protein.

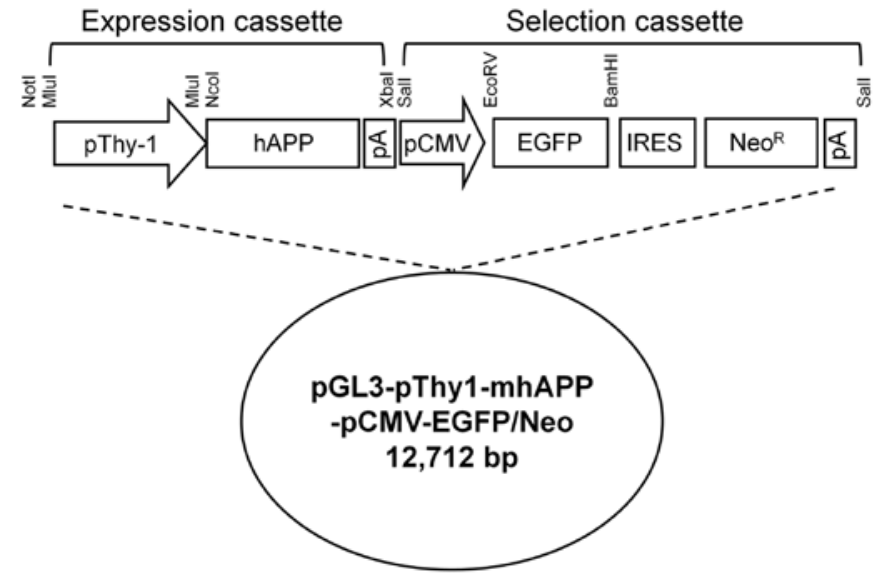

Figure 1. Schematic structure of the transgenic vector, pGL3-Thy1-mhAPPpCMV-EGFP/Neo. The transgenic vector consists of two expression and selection cassettes. The expression cassette leads to the overexpression of mutated human amyloid precursor protein $(m h A P P)$ mRNA controlled by the canine Thy-1 promoter (pThy-1). The selection cassette contains genes for enhanced green fluorescent protein (EGFP) and neomycin resistance (Neo) linked with internal ribosomal entry site (IRES) regulated by a CMV promoter (pCMV). The two cassettes were inserted into a pGL3-Basic vector.

SCNT and embryo transfer. SCNT was performed according to a previously described procedure $(5,9)$ with some modifications. Briefly, enucleation of the stripped oocytes was performed under an inverted microscope with epifluorescence (TE2000-E; Nikon Corp., Tokyo, Japan). Using an injection pipette, a trypsinized fibroblast with a smooth surface was transferred into the perivitelline space of an enucleated oocyte. The couplets were fused with two DC pulses of $1.75 \mathrm{kV} / \mathrm{cm}$ for $15 \mu \mathrm{sec}$ from a BTX Electro-Cell Manipulator 2001 (BTX Inc., San Diego, CA, USA) and transferred to naturally synchronized recipients The embryos were loaded into a Sovereign Tom Cat catheter (Sherwood Medical, St. Louis, MO, USA) with a minimal volume of medium and gently transferred into the $2 / 3$ to deep distal portion of the oviduct without insufflating air.

Diagnosis of pregnancy. Approximately 30 days after embryo transfer, the recipients were examined using a portable real-time ultrasonography machine with a $3.5-\mathrm{MHz}$ curved transducer (SSD-900; Aloka Co. Ltd., Tokyo, Japan). To detect embryonic or fetal death, sizes and shapes of the chorionic cavities along with embryonic or fetal heartbeats were examined. After confirming pregnancy, this examination was performed every seven days to monitor fetal development until birth.

Genomic DNA extraction and PCR. Canine genomic DNA was isolated using a G-DEX ${ }^{\mathrm{TM}}$ II Genomic DNA Extraction kit (Intron Biotechnology, Inc., Suwon, Korea). Genomic DNA (100 ng) or plasmid DNA (10 ng) was amplified in a 50- $\mu 1$ PCR reaction containing $1 \mathrm{U}$ of $\mathrm{LA}^{\mathrm{TM}}$ or $\mathrm{Ex}^{\mathrm{TM}}-\mathrm{Taq}$ polymerase (Takara Bio, Inc.) and $10 \mathrm{pmol}$ of specific primers. PCR was carried out for 35 cycles of denaturation for $30 \mathrm{sec}$ at $94^{\circ} \mathrm{C}$, and annealing and extension for $1-3 \mathrm{~min}$ at $68^{\circ} \mathrm{C}$ followed by a final extension for $30 \mathrm{~min}$ at $72^{\circ} \mathrm{C}$. All primers are presented in 
A

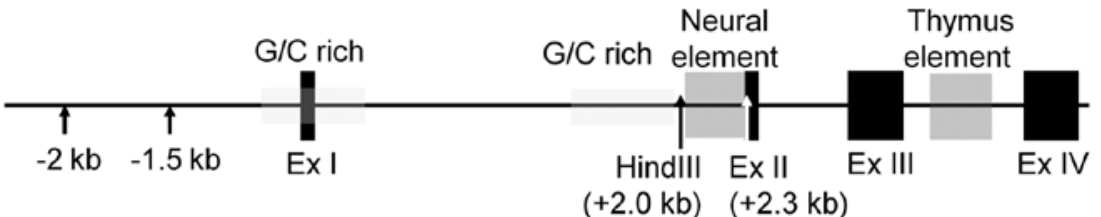

B

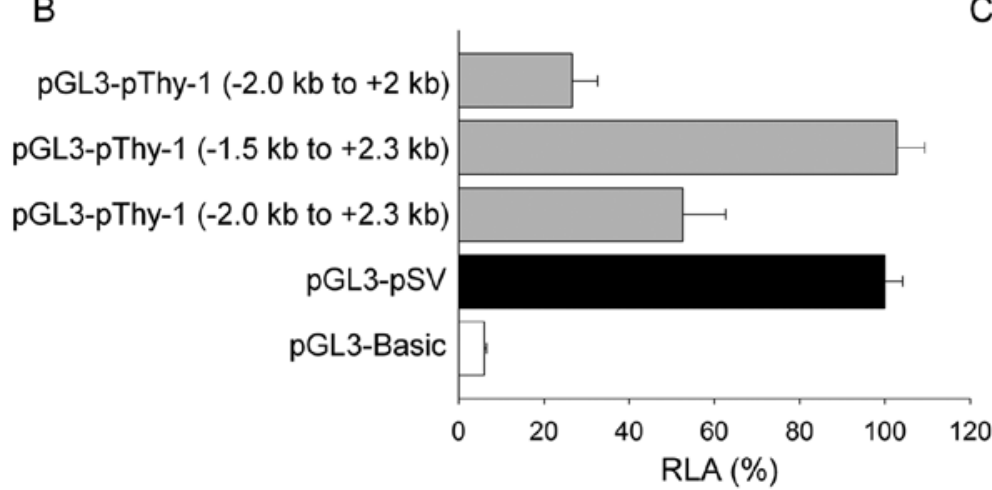

C

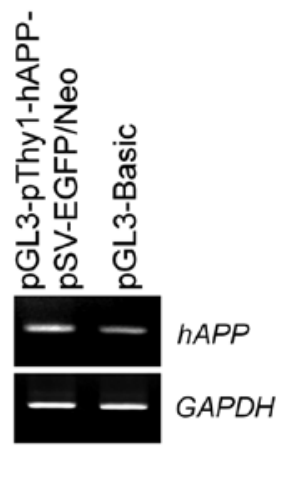

Figure 2. Canine Thy-1 promoter structure and its activities. (A) A schematic structure of the canine Thy-1 promoter region which has two tissue-specific (neurons and thymus) expression elements. (B) The promoter activities of different Thy-1 promoter regions were evaluated by a relative luciferase activities (RLA) assay. (C) The level of human amyloid precursor protein (APP) mRNA expression was measured in IMR-32 cells transiently transfected with pGL3-Thy1mhAPP-pCMV-EGFP/Neo or pGL3-Basic as a negative control. Primers specific for human APP mRNA were 5'-CAA GAC GGA GGA GAT CTC TGA-3' and 5'-CAC CAT GAT GAA TGG ATG TG-3', and generated a 212-bp polymerase chain reaction (PCR) product.

Table I. PCR products were separated on an agarose gel, stained with ethidium bromide and photographed under UV illumination. The images were scanned using a Gel Doc EQ system (Bio-Rad Laboratories, Inc., Hercules, CA, USA).

RNA preparation and quantitative RT-PCR. Total RNA was isolated from the heart, pancreas, liver, lungs, kidneys, spleen and cerebrum of two puppies (AM144 and AM145) using TRIzol reagent (Invitrogen). DNA contamination was further eliminated by treatment with DNAase (Invitrogen). Total RNA $(1 \mu \mathrm{g})$ was reverse transcribed into first-strand cDNA using M-MLV reverse transcriptase (Invitrogen) and random primers (9-mer; Takara Bio, Inc.). The GAPDH gene was amplified to evaluate RNA degradation and was used to control for variation in mRNA concentration in the RT-PCR reactions. GAPDH and human $A P P$ genes were quantified using 28 and 30 cycles, respectively. cDNA was amplified in a $20-\mu 1$ PCR reaction containing $1 \mathrm{U}$ TaqDNA polymerase (Intron Biotechnology, Inc.) and 10 pmol of the specific primers (Table I). The PCR procedure included denaturation at $95^{\circ} \mathrm{C}$ for $30 \mathrm{sec}$, annealing at $58^{\circ} \mathrm{C}$ for $30 \mathrm{sec}$ and extension at $72^{\circ} \mathrm{C}$ for $30 \mathrm{sec}$. The PCR products $(8 \mu \mathrm{l})$ were separated on a $2 \%$ agarose gel, stained with ethidium bromide and photographed under UV illumination. The images were scanned using a Gel Doc EQ system (Bio-Rad Laboratories, Inc.).

Western blot analysis. Proteins $(40 \mu \mathrm{g})$ were separated by electrophoresis on SDS-PAGE and then transferred onto a PVDF membrane. The membrane was incubated with antibodies against the following proteins: APP and A $\beta$ (1:1,000; cat. nos. 2450 and 2454; Cell Signaling Technology, Beverly, MA, USA) or $\beta$-actin (1:2,000; Santa Cruz Biotechnology, Inc., Santa Cruz, CA, USA). Immunoreactive proteins were visualized by exposure to X-ray film. Band intensities were quantified by image scanning using a Gel Doc EQ system (Bio-Rad Laboratories, Inc.), corrected by background subtraction, and normalized using $\beta$-actin as an internal control.

Immunohistochemical staining. Brain tissues were embedded in paraffin. Sections (5- $\mu \mathrm{m}$ thick) were deparaffinized and hydrated in descending grades of ethanol. The sections were subsequently incubated at room temperature for $2 \mathrm{~h}$ with an anti-A $\beta$ antibody (diluted 1:100, \#2454; Cell Signaling Technology) and rabbit anti-ionized calcium-binding adapter molecule 1 (Iba1, diluted 1:500, \#019-19741; Wako Pure Chemical Industries Ltd., Osaka, Japan) in 10\% normal goat serum. The sections were reacted with biotinylated goat antirabbit IgG (Vector ABC Elite kit; Vector Laboratories, Inc., Burlingame, CA, USA) for $45 \mathrm{~min}$ at room temperature. Immunoreactivity was assessed using the avidin-biotin peroxidase complex (Vector ABC Elite kit) prepared according to the manufacturer's instructions. The peroxidase reaction was developed using a diaminobenzidine substrate kit (SK-4100; Vector Laboratories, Inc.). The primary antibodies were omitted for a few test sections in each experiment as a control. The sections were counterstained with Harris's hematoxylin before mounting.

\section{Results}

Constructionandfunctionalactivity of the transgenic vector. We generated a human APP gene expressing a cassette containing two well-characterized mutations, Swedish (K670N and M671L) and Indiana FAD (V717F), which successfully induces AD-like pathological symptoms in mouse models (10-12). The $m h A P P$ gene was controlled by a canine $T h y-1$ promoter for selective expression in neural tissues. Specific regions of the Thy- 1 promoter were selected by a luciferase activity assay in 
A
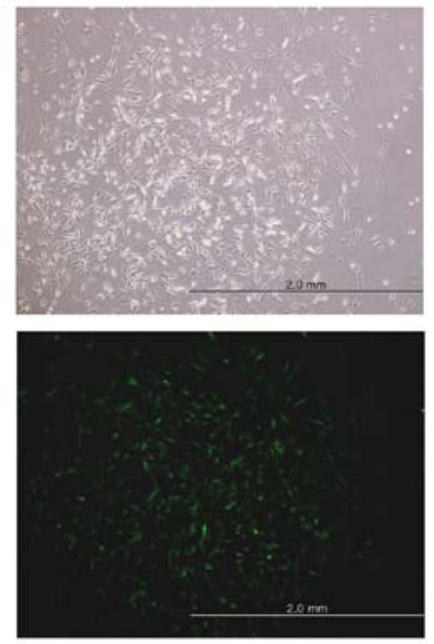

B
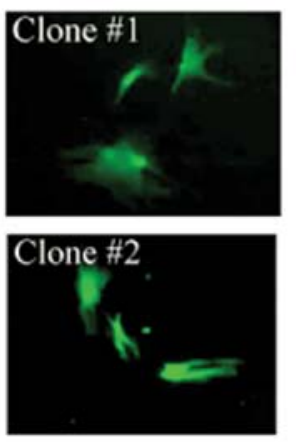

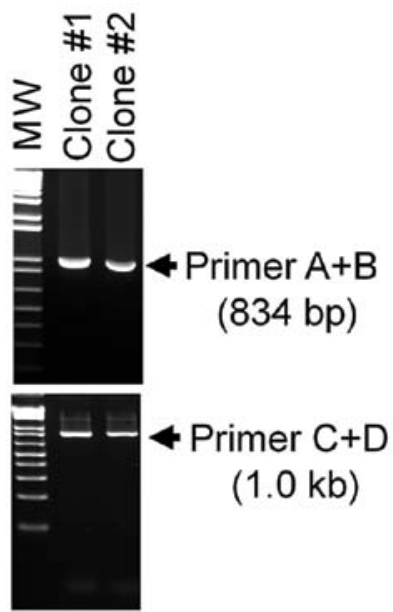

Figure 3. Confirmation of transgenic fibroblasts. Canine fibroblasts and transgenic puppies were confirmed to express enhanced green fluorescent protein (EGFP) and underwent polymerase chain reaction (PCR) analysis. (A) A representative clone of the transgenic fibroblasts was observed by light and fluorescence microscopy. Bar indicates $2 \mathrm{~mm}$. (B) PCR was carried out to analyze the two transgenic fibroblast clones. Primers A + B (834 bp) amplified the region of the selection cassette and primers $\mathrm{C}+\mathrm{D}(1 \mathrm{~kb})$ produced fragments of the expression cassette. $\mathrm{MW}$, molecular weight (1-kb ladder).

human IMR-32 neuroblastoma cells (Fig. 2A and B). We deleted the upstream portion $(-2.0$ to $-1.5 \mathrm{knts},+1=$ transcriptional start) of the Thy- 1 promoter as it possessed unknown inhibitory elements, and the neural element (between -2.0 to $-2.3 \mathrm{knts}$ ) in intron A was added as it was essential for promoter activity in the IMR-32 cells. Finally, the $T h y-1$ promoter ( -1.5 to $+2.3 \mathrm{knts})$ was found to have maximum activities similar to the SV promoter in neural cells. The transgenic vector was transiently expressed in IMR-32 cells and induced expression levels of APP transcripts two-fold higher compared to the empty pGL3-Basic Vector (Fig. 2C). The transgenic vector also contained a selection cassette including EGFP and neomycin resistance genes linked by IRES, and were controlled by the CMV promoter to facilitate selection for further processes.

Establishment and confirmation of the somatic cell line as a nuclear donor. Canine fibroblasts were transfected with the linearized transgenic vector and cultured in medium containing $350 \mu \mathrm{g} / \mathrm{ml}$ of G418 to select a transgenic clone. The cells resistant to $\mathrm{G} 418$ for four weeks were further confirmed to express EGFP by fluorescence microscopy (Fig. 3A). The insertion of the transgenic vector in the transgenic cells was confirmed by PCR (Fig. 3B). The positive cells were isolated and frozen until SCNT.

Production and characterization of transgenic puppies. SCNT was performed using the transgenic cell line and 332 oocytes matured in vivo from 29 donors. Six puppies (AM141 to 146) were born from four out of 17 surrogates. Five puppies (AM141, 142, 143, 144 and 146) were confirmed to be transgenic animals by PCR (Fig. 4A), and observed to express EGFP in their nails, toes and fur (Fig. 4B).

An EGFP-positive puppy (AM144) and EGFP-negative littermate (AM145) were sacrificed to measure $m h A P P$ expression inserted by SCNT. To eliminate genomic DNA contamination, we treated DNase I on total RNAs before the reverse-transcriptase reaction. In addition, we designed specific primers to distinguish between human and canine $A P P$ transcripts on their mRNA sequences. The expression of the $m h A P P$ gene was only observed in the organs of the EGFPpositive puppy (Fig. 4C). The amplified $m h A P P$ fragments were confirmed not to be due to genomic DNA contamination (Fig. 4D). Endogenous canine APP mRNA was detected in the organs of both animals. Amplified $m h A P P$ transcription was further confirmed by sequence analysis. Thus, the transgenic puppies we created were found to successfully express the exogenous $m h A P P$ gene.

AD-associated pathology in transgenic canines. One of the mhAPP transgenic canines (AM146) was mated with a wild-type female dog (AF165) to produce more puppies for observing the development of AD-like symptoms and stabilizing of the $m h A P P$ gene. This mating produced four male and three female puppies (SB16 to 22). All canines had the transgenic vector in their genomic DNA apart from SB21 (Fig. 5A). When the puppies were six months old, one transgenic puppy (SB17) presented recurrent behavioral disorders, such as tetanic convulsions. Although seizures are not a common phenotype of $\mathrm{AD}$, recent reports have indicated that seizures may be one of the phenotypes of AD (13-17). The brain of SB17 was further examined by magnetic resonance imaging (MRI, 7.0 T). AD-like symptoms, including enlarged ventricles and an atrophied hippocampus, were observed (Fig. 5B). The symptoms are not common in canines; however, emerging evidence has indicated that ventricle enlargement and hippocampal atrophy are phenotypes of human AD (18-21). Although the other littermates did not develop the behavioral disorders observed in SB17, an MRI image of SB19 and SB20 showed results similar to those of SB17, indicating that these puppies also had enlarged ventricles and an atrophied hippocampus (Fig. 5C and D). Our results indicated that the transgenic puppies had developed human AD-like symptoms. 
A

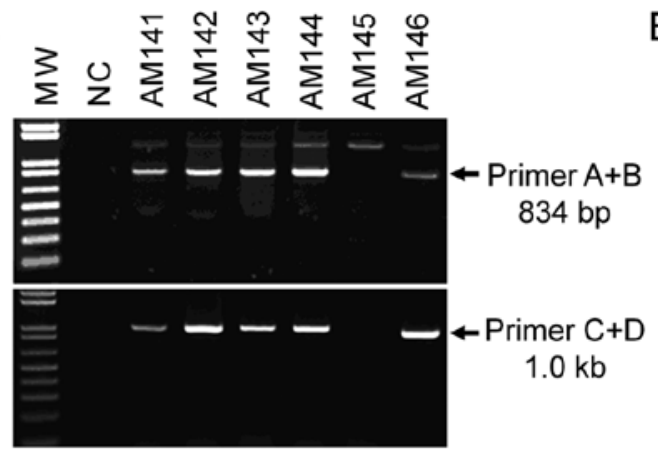

B

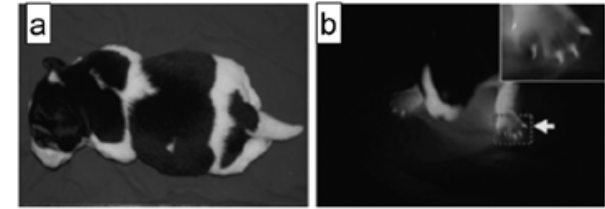

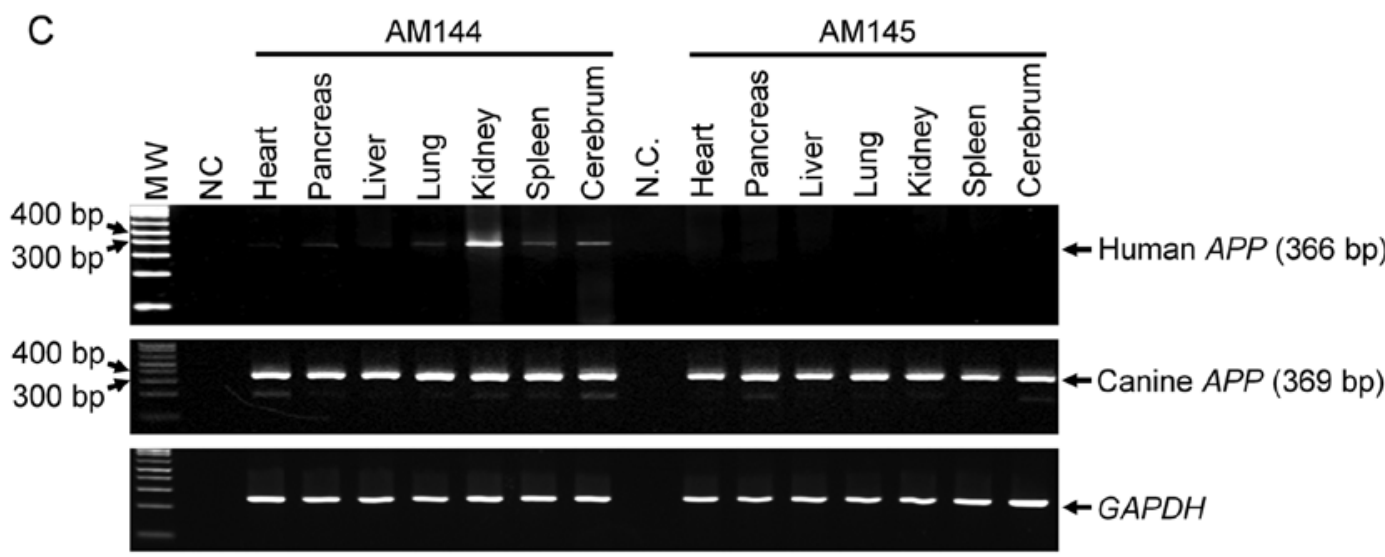

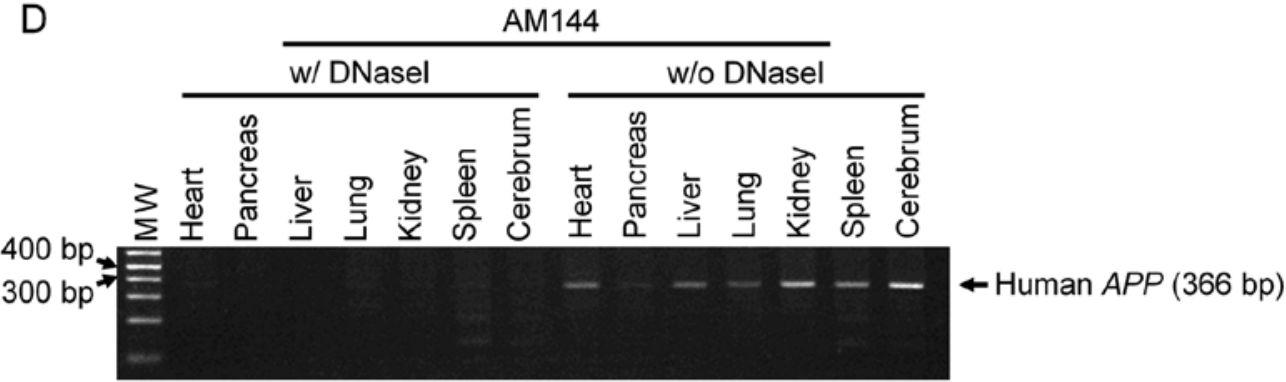

Figure 4. Confirmation of transgenic puppies with polymerase chain reaction (PCR) and enhanced green fluorescent protein (EGFP) expression. (A) Six puppies (AM141 to AM146) from the surrogates were confirmed to have undergone genetic transmission from the transgenic fibroblast with two primer sets. Primers A + B (834 bp) amplified the region of the selection cassette and primers C + D (1 kb) produced fragments of the expression cassette. MW, molecular weight; $\mathrm{NC}$, negative control (no template in the PCR reaction). (B) AM144 died $48 \mathrm{~h}$ after birth. Post-mortem examination revealed that the puppy suffered from a severe cleft palate. (a) Image from transmission light microscopy; (b) EGFP expression in the nails (dotted rectangle), toes and white fur. (C) Transgenic puppy (AM144) and its littermate (AM145) were sacrificed to measure the level of human amyloid precursor protein (APP) gene expression. Human APP (upper panel), canine APP (middle panel) and GAPDH (lower panel, internal control) gene transcription was detected in various organs by RT-PCR as indicated. (D) The same RNA samples form AM144 without RT reaction following treatment with DNase I were amplified with the same primers as in (C) to exclude genomic DNA contamination.

SB17 was sacrificed and pathological AD phenotypes in the entire brain were analyzed. The cerebral ventricles were greatly enlarged (Fig. 5E). The hippocampus had also atrophied and became degenerated in the transgenic puppy compared to the non-transgenic animal, as previously documented by an MRI. To further characterize this animal, we examined the molecular mechanisms underlying the AD-like symptoms. $A P P$ was highly expressed in the cerebrum and cerebellum of the transgenic puppy compared to the control animal (Fig. 6). However, we could not distinguish the expression of $m h A P P$ from that of endogenous canine $A P P$ with the currently available anti-APP sera. Increased levels of $A \beta$, hallmarks of AD, were also observed in the transgenic puppy. This finding suggests that the overexpression of $m h A P P$ in canines induces an accumulation of the hallmarks of $\mathrm{AD}(\mathrm{A} \beta$ accumulation in the brain, resulting in AD-like symptoms such as enlarged ventricles, an atrophied hippocampus and abnormal behavior).

For additional histopathological characterization, $A \beta$ accumulation in the hippocampal regions was further measured with an immunohistochemical assay. In the hippocampus, $A \beta$-positive immunoreactivity was rarely detected in non-transgenic canines (Fig. 7A and D), whereas the immunoreactivity was observed to a great extent in the transgenic canines (Fig. 7B and E). Additionally, microglial activation was observed in the hippocampus of the transgenic canines where $\mathrm{A} \beta$ accumulation was present (Fig. $7 \mathrm{C}$ and $\mathrm{F}$ ). These findings suggest that $A \beta$ accumulation in cells induces an immune response, as previously described (22). 
A

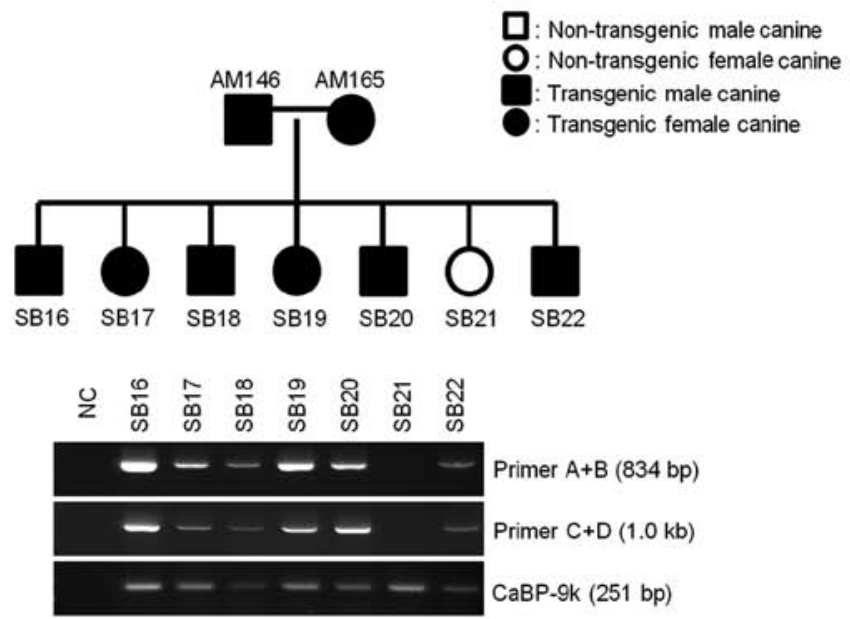

C
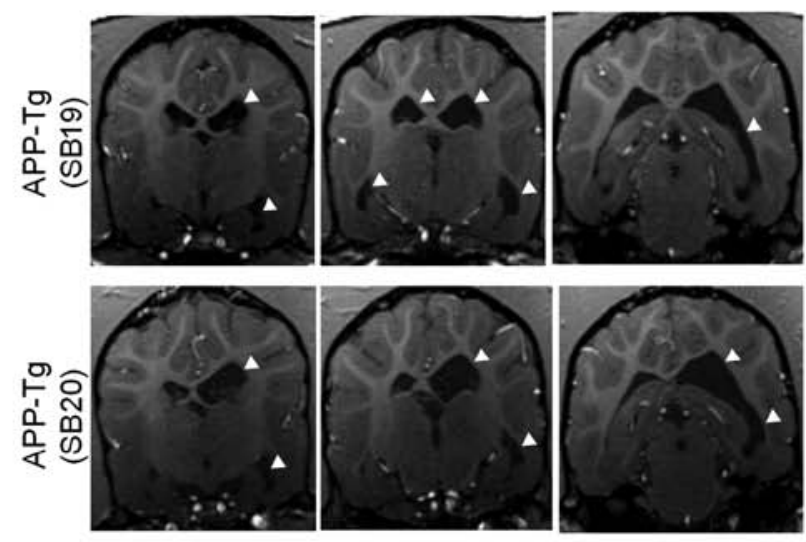

B
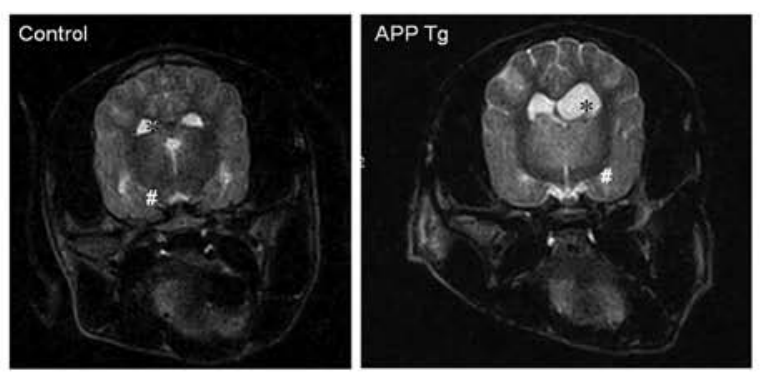

D

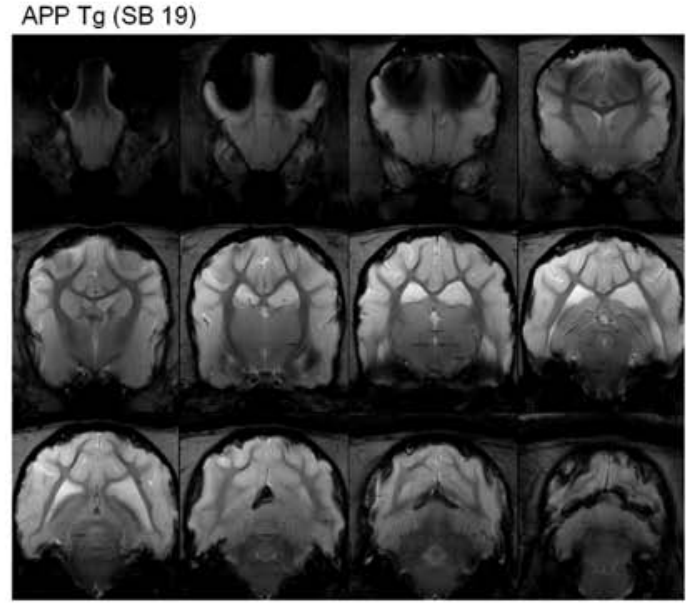

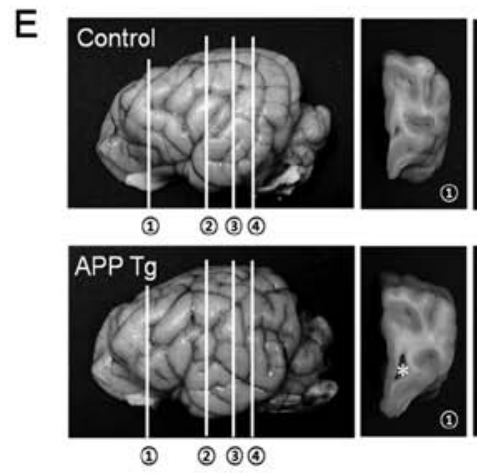
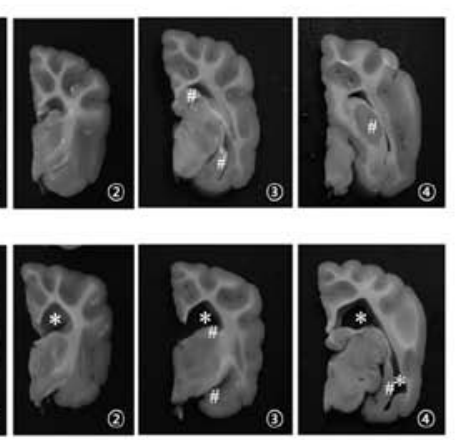

Figure 5. Alzheimer's disease (AD)-like symptoms in the transgenic canine brain. (A) Family tree and confirmation of mutated human amyloid precursor protein $(m h A P P)$ transgene integration is shown. One of the $m h A P P$ transgenic puppies (AM146, male) and a non-transgenic animal (AM165, female) were mated. Seven offspring (four males and three females) were confirmed to express the $m h A P P$ transgene with polymerase chain reaction (PCR) methods using two primer pairs. Primers A + B (834 bp) amplified the region of the selection cassette and primers C+D (1 kb) produced fragments of the expression cassette. SB17 developed abnormal behavior and was sacrificed for further study along with SB21 as a non-transgenic control. The CaBP-9k gene was amplified as an input control. NC, negative control. (B) Magnetic resonance imaging (MRI) scans of the transgenic (SB17) and non-transgenic (SB21) canines were acquired. (C) Brain MRI scans of two mhAPP transgenic canines (SB19 and SB20). White arrows indicate the enlarged ventricles. (D) Serial brain MRI scans of the transgenic canine (SB19). (E) Gross appearances of the transgenic (SB17) and non-transgenic (SB21) brains were compared. Cross-sections are indicated as circled numbers (1-4). "ventricles; "hippocampus; APP Tg, SB17; control, SB21.

\section{Discussion}

In this study, we generated transgenic canines overexpressing the human APP gene containing two well-known AD-associated mutations, Swedish (K670N and M671L) and Indiana FAD (V717F), which inhibited proteolytic processing by $\alpha$-secretase. The animals were produced with a non-viral gene delivery system to avoid unknown side-effects of viral protein contamination in the chromosome. mhAPP was successfully overexpressed in the brain of the transgenic animals and the protein end-product, $\mathrm{A} \beta$, accumulated in the brain. The transgenic canines developed abnormal neurological symptoms, such as tetanic convulsions along with enlarged ventricles and an atrophied hippocampus, similar to humans with $\mathrm{AD}$. In addition, $\mathrm{A} \beta$ plaque-like 

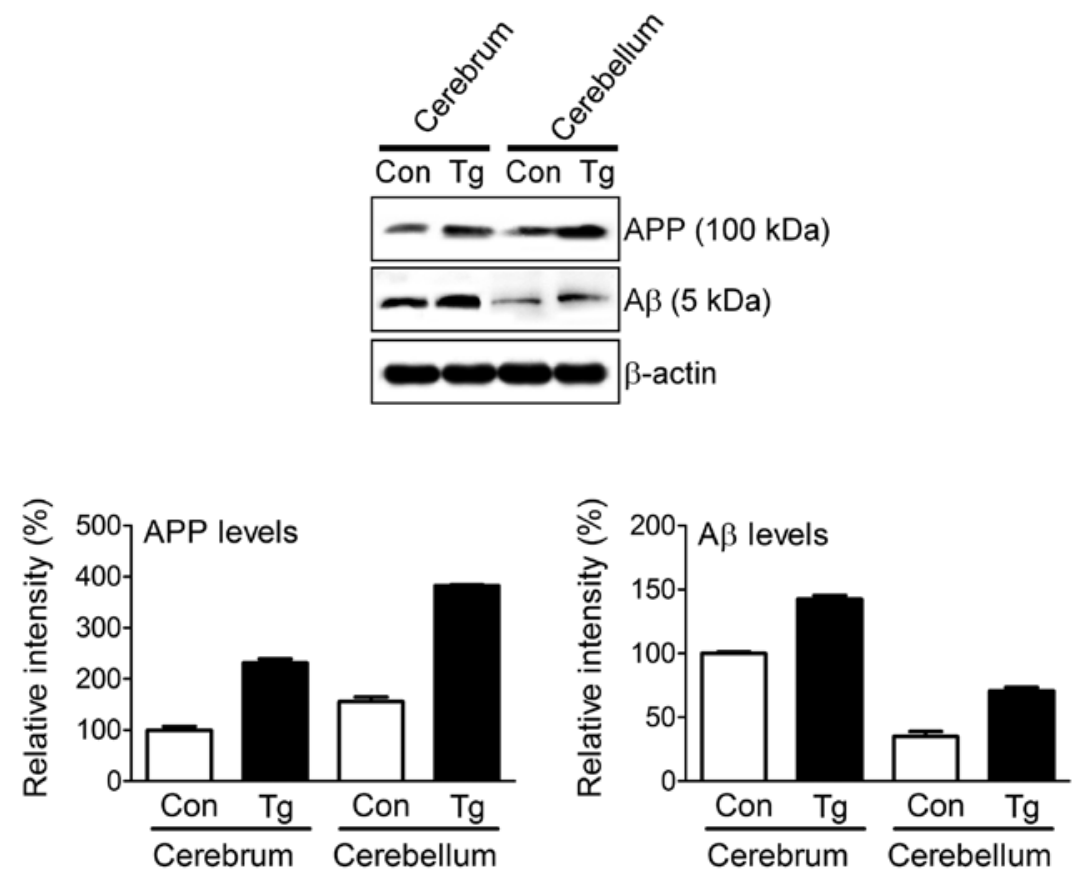

Figure 6. Confirmation of the canine modle of Alzheimer's disease (AD) by mmunoblot analysis. The transgenic canine (Tg and SB17) presenting abnormal behavior and the age-matched control (Con and SB21) were sacrificed. The expression of the amyloid precursor protein (APP) and $\beta$-amyloid (A $\beta$ ) was analyzed as indicated. The cerebrum includes the cortex region. Relative protein expression levels are presented as a graph (means \pm SEM of duplicated values from all samples).

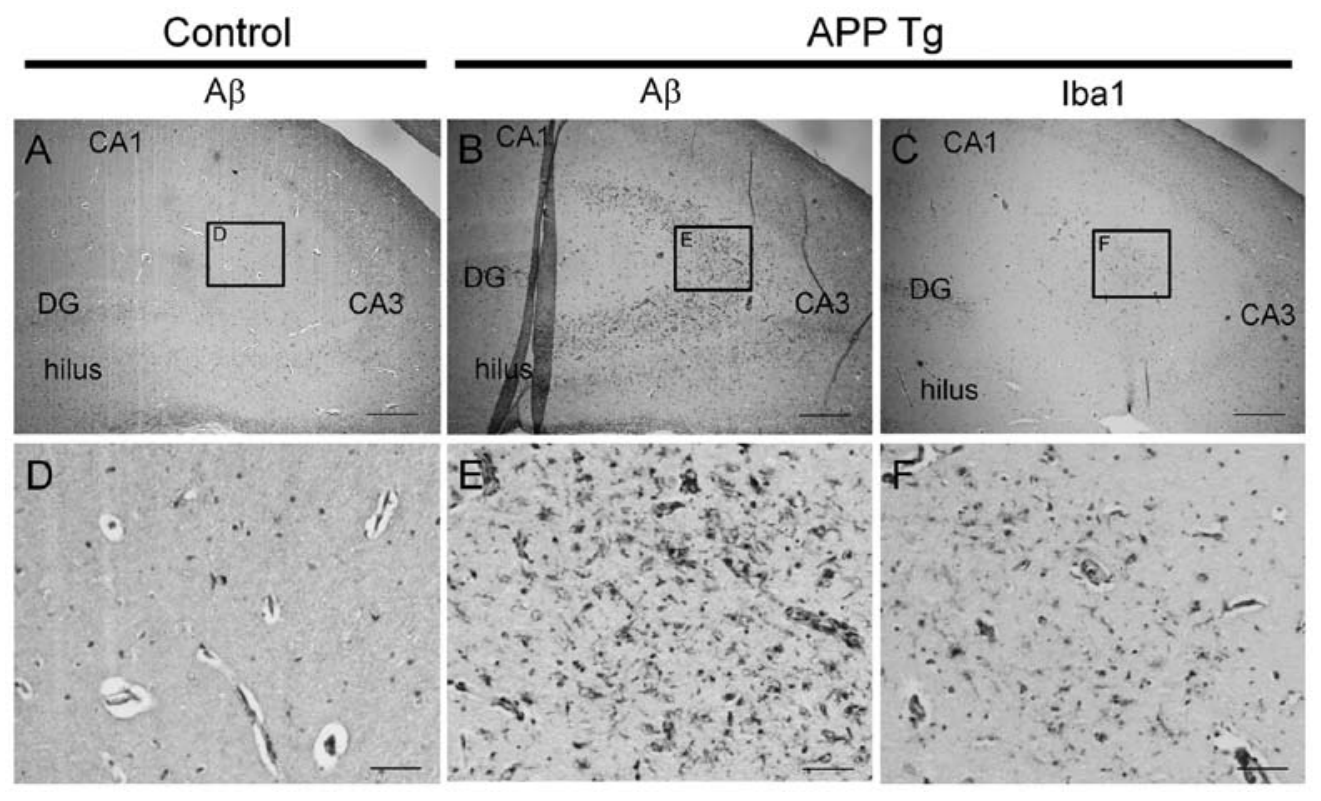

Figure 7. $\beta$-amyloid $(\mathrm{A} \beta)$ immunoreactivity in the transgenic canine. The transgenic canine [amyloid precursor protein $(A P P) \mathrm{Tg}, \mathrm{SB} 17]$ presenting abnormal behavior and the age-matched control (SB21) were sacrificed. Spatial expression of the Alzheimer's disease (AD) hallmark protein, A $\beta$, was analyzed. Thin sections were prepared from the hippocampal region. The (A and D) left and (B and E) middle panels indicate A $\beta$-specific staining. The $(\mathrm{C}$ and $\mathrm{F})$ right panels indicate the staining for ionized calcium-binding adapter molecule 1 (Iba1), a microglial marker, using the serial section of the middle panels (B and E). Scale bars: (A-C) $300 \mu \mathrm{m}$, (D-F) $80 \mu \mathrm{m}$. CA, Cornu Ammonis; DG, dentate gyrus.

structures, a histopathological hallmark of $\mathrm{AD}$, were detected in the frontal cortex regions, and significant $A \beta$ accumulation was further observed in the cortex and hippocampus. Thus, the transgenic canines we produced are good candidates for replacing the present rodent model for studying the pathophysiological characteristics of $\mathrm{AD}$ and performing pharmaceutical research for the treatment of this disease.
$\mathrm{AD}$ is the most common dementia disorder in humans. The major pathological hallmarks of this disease are an abnormal accumulation of extracellular amyloid plaques and intracellular NFTs in vulnerable brain regions. The tangles are primarily composed of paired helical filaments of hyperphosphorylated forms of the microtubule-associated protein, tau. The main constituent of the plaques is $\mathrm{A} \beta$, a peptide with 
40-42 amino acids derived from APP by sequential proteolytic cleavage by $\beta$ - and $\gamma$-secretase (23-25). Numerous mouse lines that develop A $\beta$ deposits have been produced (6). MhAPP transgenes in mice cause $A \beta$ deposition, although these lesions are not identical to the $A \beta$ protein in human $A D$ plaques. Aside from $\mathrm{A} \beta$ deposition, axon dystrophy and dendrite alterations have been observed (6). All APP mutations in transgenic mice increase $A \beta 42$ levels, although the overexpression of wildtype APP alone does not induce A $\beta$ deposition in mice (6). Double transgenic mice (expressing both $A P P$ and $P S 1$ ) develop lesions earlier. The development of $A P P$ transgenic mice has raised new questions concerning the mechanisms of neuronal loss, the accumulation of $\mathrm{A} \beta$ protein in the body of neurons, inflammation and gliosis, and dendritic alterations. These data from transgenic mice have provided some insight into the kinetics of the pathogenesis of AD. The connections between $\mathrm{AD}$-associated symptoms, lesions and increased levels of $A \beta$ oligomers have been difficult to unravel. Thus far, the best animal model for studying AD has been transgenic mice. However, the transgenic canines presenting AD-like symptoms we generated may prove to be useful for studying $\mathrm{AD}$ in humans.

Successful studies of human diseases require an appropriate animal model. Common animal models for AD research are transgenic mice that overexpress a mutant form of the human $\mathrm{A} \beta$ precursor protein and/or enzymes implicated in its metabolic processing (26). However, rodent models are not sufficient to fully elucidate the pathogenesis of AD due to genetic, physiological and anatomic differences between mice and humans. Canines are more suitable for examining human disorders than mice, as canines have evolved physiologically and genetically in close proximity to humans $(27,28)$. In addition, canine models naturally develop an age-related cognitive dysfunction that reproduces several aspects of $\operatorname{AD}(27,28)$. Numerous studies using canine cohorts examining several behavioral paradigms have revealed subsets of aged canines with learning and memory impairments (29-31). Thus, canine models have been identified as a unique model for studying a human disorders, such as AD.

Although a canine model is ideal for human disease studies, to our knowledge, no such model has been reported to date, due to the lack of canine embryonic stem cells, which are generally used for gene targeting, and proper protocols for producing mature oocytes in vitro. From the molecular point of view, the canine is a suitable AD model animal as APP and most of the enzymatic machinery for processing this factor are highly homologous in canines and humans. This genetic similarity allows us to expect concordance in gene expression and development of the disease phenotype in the transgenic puppies. This may facilitate fundamental studies of the disease process and phenotype development as a function of time.

In a previous study, Hong et al (7) reported the production of a transgenic canine expressing red fluorescent protein using retroviral gene delivery methods. Although the efficient genetic modification of donor cells is a key prerequisite to produce transgenic animals, viral delivery systems are associated a minor risk of neoplastic transformation (32). To prevent these unwanted effects, in this study, we generated five genetically engineered puppies expressing $m h A P P$ using a liposomal transfection method. The stable transfection of canine fibroblasts with a liposomal reagent is a very effective method that can replace viral gene delivery techniques. In this study, the expression and selection cassettes were successfully integrated into genomic DNA and effectively expressed in tissues following SCNT. This non-viral, SCNT-mediated gene delivery system is a safer protocol for creating animal disease models than viral gene delivery.

Canines naturally develop AD and senile plagues similar to humans, and the plaques are initially observed between the ages of eight and nine years (33). The pathological features in aged canines having AD-like symptoms are quite similar to human phenotypes, such as neuronal loss and cognitive deficits. These progressive studies suggest that canines are the best model for studying human AD. Although the aged canine model has several advantages, the fact that over eight years are required to develop $\mathrm{AD}$ symptoms is still a major obsticle for general usage in the research field. Hence, we suggest the use of transgenic canines carrying $m h A P P$ to induce the early onset of $\mathrm{AD}$ phenotypes, as performed in the present study.

In conclusion, we generated a canine model of AD to replace the current rodent models. This is important as canines possess a higher intelligence than mice and are large enough to undergo surgical procedures in pre-clinical studies. This canine model expressing the $m h A P P$ gene produced by SCNT may be used for understanding the pathological and developmental characteristics of AD, and may aid in the development of novel therapeutic modalities for AD.

\section{Acknowledgements}

We thank Zang-Hee Cho and Young-Bo Kim (Neuroscience Research Institute, Gachon University of Medicine and Science, Incheon, Republic of Korea) for helping with the analyses of the brain MRI scans of the transgenic canines. This study was supported by grants from the Next-Generation BioGreen 21 Program [nos. PJ008323 and PJ009489], Rural Development Administration (RDA), Republic of Korea.

\section{References}

1. Rocchi A, Pellegrini S, Siciliano G and Murri L: Causative and susceptibility genes for Alzheimer's disease: a review. Brain Res Bull 61: 1-24, 2003.

2. Götz J, Streffer JR, David D, et al: Transgenic animal models of Alzheimer's disease and related disorders: histopathology, behavior and therapy. Mol Psychiatry 9: 664-683, 2004.

3. Head E, Pop V, Sarsoza F, et al: Amyloid-beta peptide and oligomers in the brain and cerebrospinal fluid of aged canines. J Alzheimers Dis 20: 637-646, 2010.

4. Kragh PM, Nielsen AL, Li J, et al: Hemizygous minipigs produced by random gene insertion and handmade cloning express the Alzheimer's disease-causing dominant mutation APPsw. Transgenic Res 18: 545-558, 2009.

5. Jang G, Kim MK, Oh HJ, et al: Birth of viable female dogs produced by somatic cell nuclear transfer. Theriogenology 67 : 941-947, 2007.

6. Duyckaerts C, Potier MC and Delatour B: Alzheimer disease models and human neuropathology: similarities and differences. Acta Neuropathol 115: 5-38, 2008.

7. Hong SG, Kim MK, Jang G, et al: Generation of red fluorescent protein transgenic dogs. Genesis 47: 314-322, 2009.

8. Hossein MS, Kim MK, Jang G, et al: Effects of thiol compounds on in vitro maturation of canine oocytes collected from different reproductive stages. Mol Reprod Dev 74: 1213-1220, 2007.

9. Lee BC, Kim MK, Jang G, et al: Dogs cloned from adult somatic cells. Nature 436: 641, 2005. 
10. Spanopoulou E, Giguere V and Grosveld F: The functional domains of the murine Thy- 1 gene promoter. Mol Cell Biol 11: 2216-2228, 1991

11. Dudal S, Krzywkowski P, Paquette J, et al: Inflammation occurs early during the Abeta deposition process in TgCRND8 mice. Neurobiol Aging 25: 861-871, 2004.

12. Spires TL and Hyman BT: Transgenic models of Alzheimer's disease: learning from animals. NeuroRx 2: 423-437, 2005.

13. Friedman D, Honig LS and Scarmeas N: Seizures and epilepsy in Alzheimer's disease. CNS Neurosci Ther 18: 285-294, 2012.

14. Irizarry MC, Jin S, He F, et al: Incidence of new-onset seizures in mild to moderate Alzheimer disease. Arch Neurol 69: 368-372, 2012.

15. Larner AJ and Marson AG: Epileptic seizures in Alzheimer's disease: another fine MESS? J Alzheimers Dis 25: 417-419, 2011

16. Pandis D and Scarmeas N: Seizures in Alzheimer disease: clinical and epidemiological data. Epilepsy Curr 12: 184-187, 2012.

17. Picco A, Archetti S, Ferrara M, et al: Seizures can precede cognitive symptoms in late-onset Alzheimer's disease. J Alzheimers Dis 27 737-742, 2011.

18. Apostolova LG, Green AE, Babakchanian S, et al: Hippocampal atrophy and ventricular enlargement in normal aging, mild cognitive impairment (MCI), and Alzheimer Disease. Alzheimer Dis Assoc Disord 26: 17-27, 2012.

19. Chou YY, Leporé N, Avedissian C, et al: Mapping correlations between ventricular expansion and CSF amyloid and tau biomarkers in 240 subjects with Alzheimer's disease, mild cognitive impairment and elderly controls. Neuroimage 46: 394-410, 2009.

20. Ferrarini L, Palm WM, Olofsen H, et al: MMSE scores correlate with local ventricular enlargement in the spectrum from cognitively normal to Alzheimer disease. Neuroimage 39: 1832-1838, 2008.

21. Nestor SM, Rupsingh R, Borrie M, et al: Ventricular enlargement as a possible measure of Alzheimer's disease progression validated using the Alzheimer's disease neuroimaging initiative database. Brain 131: 2443-2454, 2008.
22. Glass CK, Saijo K, Winner B, Marchetto MC and Gage FH: Mechanisms underlying inflammation in neurodegeneration. Cell 140: 918-934, 2010

23. Dyrks T, Weidemann A, Multhaup G, et al: Identification, transmembrane orientation and biogenesis of the amyloid $\mathrm{A} 4$ precursor of Alzheimer's disease. EMBO J 7: 949-957, 1988.

24. Selkoe DJ: Alzheimer's disease: genes, proteins, and therapy. Physiol Rev 81: 741-766, 2001.

25. Sisodia SS: Beta-amyloid precursor protein cleavage by a membrane-bound protease. Proc Natl Acad Sci USA 89: 6075-6079, 1992.

26. Sarasa M and Pesini P: Natural non-trasgenic animal models for research in Alzheimer's disease. Curr Alzheimer Res 6: 171-178, 2009.

27. Head E: Combining an antioxidant-fortified diet with behavioral enrichment leads to cognitive improvement and reduced brain pathology in aging canines: strategies for healthy aging. Ann NY Acad Sci 1114: 398-406, 2007.

28. Siwak-Tapp CT, Head E, Muggenburg BA, Milgram NW and Cotman CW: Region specific neuron loss in the aged canine hippocampus is reduced by enrichment. Neurobiol Aging 29: 39-50, 2008.

29. Head E, Mehta R, Hartley J, et al: Spatial learning and memory as a function of age in the dog. Behav Neurosci 109: 851-858, 1995.

30. Adams B, Chan A, Callahan $\mathrm{H}$, et al: Use of a delayed nonmatching to position task to model age-dependent cognitive decline in the dog. Behav Brain Res 108: 47-56, 2000.

31. Smith DR, Hoyt EC, Gallagher M, Schwabe RF and Lund PK Effect of age and cognitive status on basal level AP-1 activity in rat hippocampus. Neurobiol Aging 22: 773-786, 2001.

32. Sokol DL and Gewirtz AM: Gene therapy: basic concepts and recent advances. Crit Rev Eukaryot Gene Expr 6: 29-57, 1996.

33. Martin SB, Dowling AL and Head E: Therapeutic interventions targeting Beta amyloid pathogenesis in an aging dog model. Curr Neuropharmacol 9: 651-661, 2011. 